\title{
A new assessment method based on fuzzy linguistic of Engineering bidding risk prediction model
}

\author{
Shumei Cui \\ Civil Engineering Institution, Yantai University, China \\ 13954524999@163.com
}

\begin{abstract}
Keywords: Engineering bidding; risk factors; multi-attribute decision-making; fuzzy linguistic; the method of variation coefficient; project bidding risk assessment model; project example
\end{abstract}

\begin{abstract}
Bid risk is the important factors considered by the bidders in bid decision-making process. And bid risk analysis is a typical multi-attribute decision-making problem. Therefore, this paper proposes a new bid risk prediction model, based on the bidders' risk prediction information in selecting bid project process, with multiple attribute related theory on fuzzy linguistic for tools. A simple and explicit formula for obtaining the rick factors' weights is got by the concept of range extremity difference. Finally, an example is given to show the rationality and effectiveness of the new method.
\end{abstract}

\section{Introduction}

Before bidding, the bidders must investigate the bid project and then they can decide bid or not. To make the best decision and decide which project to bid, bidders should take subjective and objective factors into account, such as contractor's abilities, competitors' strengths and owners' credit. Actually, bidders indeed know their own abilities more but know less about those factors which affects the bid decision-making and the only way for them to know the factors is investigating deeply and widely but they don't have the $100 \%$ of trust in the investigation for the investigation itself exists risks. Therefore, in prophase decision-making process of bidding, the most significant task is how to avoid risks efficiently ${ }^{[1,2]}$.

Bid risk assessment of construction project is a typical multi-attribute decision-making problem ${ }^{[3,}$

${ }^{4]}$. In bid decision-making process, bidders are often required to provide risk attribute weights and assessment information of bid project in advance and then they sort every project risk with utilizing the comprehensive attribute assessment information of each bid project. However, as complexity of objective things and fuzziness of human thinking, when evaluating each qualitative factor of the tendering project, bidders are likely to describe risks with such fuzzy linguistic as 'high', 'relatively high', 'medium', 'relatively low', 'low', etc.. Hence, there are significantly theoretical meanings and highly practical values in the research of risk assessment with fuzzy linguistic which gradually draw our attentions. Therefore, as the risk attribute weights of bid project are totally unknown and based on bid risk decision-making problem in which the attribute values are given in fuzzy linguistic, this paper proposes a new bid risk decision-making model and provides a new thinking for bidders in bid risk assessment. In this model, a simple and explicit formula for obtaining the rick factors' weights is got by the concept of range extremity difference. Now we fist analyze the risk factors of bidding project.

\section{Risk factors analysis of bid project}

To predict risk efficiently, bidders generally classify risk factors in bidding process as Fig. 1 shows ${ }^{[5]}$.

Through Fig. 1, we clearly know that the risk factors should be considered when bidding and then we propose a new bid risk decision-making model based on fuzzy multi-attribute decision-making theory. 


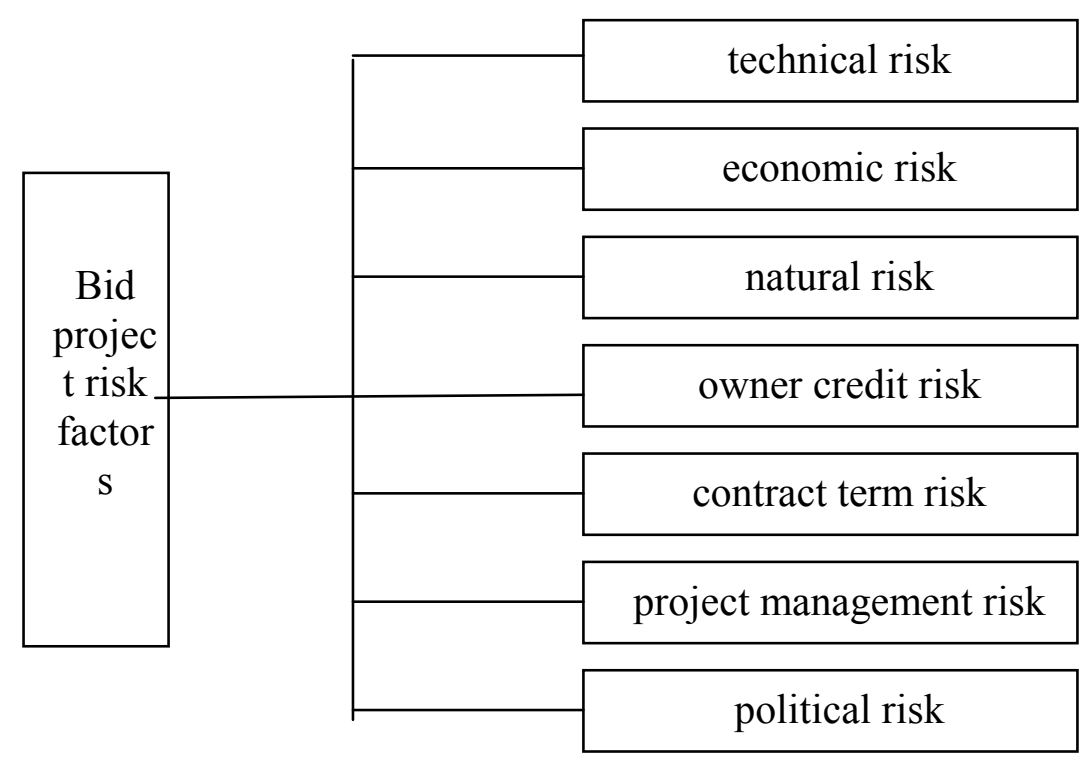

Fig 1 bidding risk factor classification

\section{Problems and Analysis}

In regard to the multi-attribute decision-making problem of bid risk assessment, supposing $X=\left\{x_{1}, x_{2}, \cdots, x_{n}\right\}$ stands for bid project set, $G=\left\{f_{1}, f_{2}, \cdots, f_{m}\right\}$ stands for risk factors attribute set. $y_{i j}=f_{i}\left(x_{j}\right)(i=1,2 \cdots ; m j=1,2 \cdots ; n)$ is the fuzzy linguistic attribute value of the bid project ${ }^{x_{j}}$ under the attribute $f_{i}$, and supposing $Y=\left(y_{i j}\right)_{m \times n}$ is the evaluation matrix, furthermore $y_{i j} \in S$. In general case, the fuzzy linguistic scale $S$ can be divided into \{high, relatively high, medium, relatively low, low . The interval numbers which is relative to the scale can be shown high $(0.8,0.9,1)$; relatively high : $(0.6,0.7,0.8)$; medium : $(0.4,0.5,0.6)$; relatively low: $(0.2,0.3,0.4)$;low: $(0.0 .1 .0 .2)$.

With these conditions, we sort the risks of scheme in $X$.

Based on the thought of the difference maximum of attribute value, some decision making models are put forward in references [6]. These models can better deal with the decision making problem that attribute values are shown in the real number form. Besides, these models relate to variance calculation and deviation calculation. The concept of variance and deviation reflect the dispersion degree of attribute value.

To be precise, because of the particularity of fuzzy linguistic, the fuzzy multi-attribute decision making model is difficult to bear complex computations. Therefore, those methods proposed in references [6] are not reasonable to give attribute values in fuzzy linguistic. As to the analysis above, we make the range extremity difference whose calculated amount is small replace the variance or deviation in order to reflect the dispersion degree of a group of fuzzy language. And build a decision model which is based on the theory of range extremity difference.

\section{Decision model}

Based on the above analysis, using the evaluation criteria of fuzzy linguistic and the possibility of fuzzy number comparing formulas, this article proposes a new method of assessment the risk of bid projects based on multi-attribute decision making theory, specific steps as follows ${ }^{[7]}$ :

First step, blur matrix $Y=\left(y_{i j}\right)_{m \times n}$, then we can get a fuzzy number matrix. We may as well set the matrix as $Z=\left(z_{i j}\right)_{m \times n}$; 
Second step, take $Z_{i}(i=1,2, \cdots, m)$ as norm of every row vector in $Z=\left(z_{i j}\right)_{m \times n}$. We set norm $D_{i}$ as maximum of attribute value range $\left\|y_{i j}-y_{i k}\right\|,(1 \leq j, k \leq n)$ of the chosen vector. We use it to reflect the weight of value $f_{i}$ in the process of decision.

$D_{i}=\max \left\|y_{i j}-y_{i k}\right\|,(1 \leq j, k \leq n)$

Third step, we composite all $D_{i}$ taken from last step as an ordered array $D=\left(D_{1}, D_{2}, \cdots, D_{m}\right)_{\text {which }}$ has $m$ elements. Through $D$ normalization processing, we can get risk attribute weight vector W.

Fourth step, multiply attribute weight vector $W$ with $Z=\left(z_{i j}\right)_{m \times n}$, let be $r_{i j}=w_{i} z_{i j}$, we get weighted suitability decision matrix $R=\left(r_{i j}\right)_{m \times n}$.

Fifth step, we conduct bid risk decision according to different standards. There are many references about this step, so we don't describe it detailed.

\section{Application example}

Through the bidding decision of one construction enterprise in Yantai, we prove the effectively of the model. At the same time, there are five bid projects to be chosen. And the construction enterprise has past the prequalification. The risk factors which are considered in this bid decision are shown in fig. 1. The bidders are likely to describe risks with such fuzzy linguistic as 'high(simplified as H)', 'relatively high(RH)', 'medium(M)', 'relatively low(RL)', 'low(L)' five grades. According to the situations by invested, the risk factors' grades are shown in table 1.

Table 1 the details of the risk factors' grades for each bid

\begin{tabular}{|c|c|c|c|c|c|c|c|}
\hline $\begin{array}{c}\text { Bid } \\
\text { projec } \\
\text { t }\end{array}$ & $\begin{array}{c}\text { Technica } \\
\text { Risk }\end{array}$ & $\begin{array}{c}\text { Economic } \\
\text { Risk }\end{array}$ & $\begin{array}{c}\text { Natura } \\
1 \\
\text { Risk }\end{array}$ & $\begin{array}{c}\text { owner } \\
\text { credit } \\
\text { risk }\end{array}$ & $\begin{array}{c}\text { contrac } \\
\text { t term } \\
\text { risk }\end{array}$ & $\begin{array}{c}\text { project } \\
\text { management } \\
\text { risk }\end{array}$ & $\begin{array}{c}\text { politica } \\
1 \text { risk }\end{array}$ \\
\hline A & M & L & RL & M & M & RL & L \\
\hline B & RL & RL & RH & L & RL & M & L \\
\hline C & L & M & RL & M & M & RL & L \\
\hline D & RL & RL & M & RH & RL & RL & L \\
\hline
\end{tabular}

First step, as for the table 1, we quantize its qualitative indexes according to the modified bipolar scaling methods. We get the fuzzy index value matrix $\mathrm{Z}$.

$$
Z=\left[\begin{array}{l}
(0.4,0.5,0.6)(0,0.1,0.2)(0.2,0.3,0.4)(0.4,0.5,0.6)(0.4,0.5,0.6)(0.2,0.3,0.4)(0,0.1,0.2) \\
(0.2,0.3,0.4)(0.2,0.3,0.4)(0.6,0.7,0.8)(0,0.1,0.2)(0.2,0.3,0.4)(0.4,0.5,0.6)(0,0.1,0.2) \\
(0,0.1,0.2)(0.4,0.5,0.6)(0.2,0.3,0.4)(0.4,0.5,0.6)(0.4,0.5,0.6)(0.2,0.3,0.4)(0,0.1,0.2) \\
(0.2,0.3,0.4)(0.2,0.3,0.4)(0.4,0.5,0.6)(0.6,0.7,0.8)(0.2,0.3,0.4)(0.2,0.3,0.4)(0,0.1,0.2)
\end{array}\right]
$$

Second and third steps, use the formula (1) to calculate the weights of the risk attribute, and then normalize them to get 1 by adding them.

$$
\begin{aligned}
& w_{1}=(0.18,0.18,0.18) ; w_{2}=(0.18,0.18,0.18) ; w_{3}=(0.18,0.18,0.18) ; w_{4}=(0.27,0.27,0.27) ; \\
& w_{5}=(0.09,0.09,0.09) ; w_{6}=(0.09,0.09,0.09) ; w_{7}=(0,0,0)
\end{aligned}
$$

Fourth step, we calculate the fuzzy weighted decision matrix to get the result R. 


$$
\begin{aligned}
& R=\left[\begin{array}{l}
(0.07,0.09,0.11)(0,0.02,0.04)(0.04,0.05,0.07) \\
(0.04,0.05,0.07)(0.04,0.05,0.07)(0.11,0.13,0.14) \\
(0,0.02,0.04)(0.07,0.09,0.11)(0.04,0.05,0.07) \\
(0.04,0.05,0.07)(0.04,0.05,0.07)(0.07,0.09,0.11)
\end{array}\right. \\
& (0.11,0.14,0.16)(0.04,0.05,0.05)(0.02,0.03,0.04)(0,0,0) \\
& (0,0.03,0.05)(0.02,0.03,0.04)(0.04,0.05,0.05)(0,0,0) \\
& (0.11,0.14,0.16)(0.04,0.05,0.05)(0.02,0.03,0.04)(0,0,0) \\
& (0.16,0.19,0.22)(0.02,0.03,0.04)(0.02,0.03,0.04)(0,0,0)
\end{aligned}
$$

Fifth step, we calculate the comprehensive fuzzy evaluation matrix to get the result $\mathrm{B}$.

$$
B=[(0.28,0.38,0.47),(0.25,0.34,0.42),(0.28,0.38,0.47),(0.35,0.44,0.55)]
$$

According to the last result we sort the projects risk: $D>C=A>B$

\section{Conclusion}

It can be seen from the above examples, the bid risk decision model given by this paper for indeterminate multi-attribute decision problem, in which the attribute weights information is precisely unknown and the attribute values were given in the forms of fuzzy linguistic assessments.

A simple and explicit formula for obtaining each risk factor attribute weights is given by this paper; therefore, it has extensive promotion value. However, every method must have its shortcomings and vulnerabilities; we hope that this method can be gradually improved in practice.

\section{Acknowledgements}

This work was financially supported by the Natural Science Fund of Shandong Province (ZR2009FM074), and the Yantai University Youth Fund (TM08Z1).

\section{References}

[1] Yi Zhang: Technology EconomicsVol. 28 (2009), p. 104.

[2] Stephane Airiau and Sandip Sen: Group Decision and Negotiation, Vol. 12(2003), p. 397.

[3] Thomas Keller and Sebastian Kupferschmid:Lecture Notes in Computer Science,Vol4(2008) ), p. 95.

[4] Glasserman P and Heidelberger P: Mathematical Finance, Vol. 12(2002), p. 239.

[5] Lizhong She. Construction Project Contract Management (Third Edition), edited by South china University of Technology Press, Guangzhou, NY (2010).

[6] Zeshui Xu and Xiaoqiang Cai:.Fuzzy Optimization and Decision Making, Vol. 9 (2010), p. 359.

[7] Guangwu Meng and Xingfang Zhang: Fuzzy Systems and Mathematics, Vol. 3 (2001), p. 25. 